«Pascal Écrivain des plus eloquans » un manuscrit inédit de l'Abbé de Saint-Pierre

\title{
Antony McKenna
}

\section{CpenEdition}

\section{Journals}

Édition électronique

URL : http://journals.openedition.org/ccibp/624

DOI : $10.4000 /$ ccibp. 624

ISSN : 2493-7460

Éditeur

Centre international Blaise Pascal

Édition imprimée

Date de publication : 7 janvier 1991

Pagination : 4-7

ISSN : 0249-6674

\section{Référence électronique}

Antony McKenna, « «Pascal Écrivain des plus eloquans » un manuscrit inédit de l'Abbé de Saint-Pierre », Courrier du Centre international Blaise Pascal [En ligne], 13 | 1991, mis en ligne le 08 janvier 2016, consulté le 01 mai 2019. URL : http://journals.openedition.org/ccibp/624 ; DOI : 10.4000/ccibp.624

Ce document a été généré automatiquement le 1 mai 2019.

Centre international Blaise Pascal 


\title{
« Pascal Écrivain des plus eloquans » un manuscrit inédit de l'Abbé de Saint-Pierre
}

\author{
Antony McKenna
}

\section{Introduction}

1 Vers 1680 l'abbé de Saint-Pierre (1658-1743) arrive à Paris et retrouve ses anciens camarades du collège de Rouen : avec Fontenelle, Vertot et Varignon il constitue, dans le faubourg Saint-Jacques, une petite « académie » que fréquente Malebranche. Leurs goûts sont surtout scientifiques, mais, vers 1683, l'abbé de Saint-Pierre rompt avec la physique pour s'intéresser à la morale :

2 «Le principal goût qui m'était resté de la lecture des ouvrages de Descartes et des cartésiens au sortir du collège était pour l'étude de la physique, et comme par la mort d'un de mes parents j'étais demeuré maître de suivre mes goûts, je me livrai avec plaisir durant trois ou quatre ans à cultiver cette science [...] Mais en lisant les pensées morales de Pascal, je compris que les progrès que je pourrais faire dans la morale seraient plus utiles pour augmenter mon bonheur et le bonheur de ceux avec qui j'aurais à vivre ; aussi je quittai la physique pour étudier et méditer sur les réflexions de morale, et j'écrivais tous les jours quelques réflexions détachées soit pour ma propre instruction, soit pour celle des autres ${ }^{1}$. "

3 Pascal joue donc un rôle capital dans la vocation de l'abbé de Saint-Pierre, et, avouons-le, c'est là une lourde responsabilité, car celui-ci allait devenir non seulement un moraliste mais aussi un moralisateur intarissable, dont les projets et les rêves d'une société vertueuse et d'une vertu utile allaient entraîner l'exil.

4 Quelques années plus tard, vers 1690, Pierre Nicole confiait à ses amis ses souvenirs de Port-Royal: Jean Racine et l'oratorien Nicolas-Marie Ruffin recueillent quelques anecdotes, et c'est à cette occasion que l'abbé de Saint-Pierre entend Nicole traiter Pascal 
de « ramasseur de coquilles ». Certes, il se méprend sur le sens de cette remarque ${ }^{2}$, mais signalons surtout la complaisance avec laquelle il relève les prétendues faiblesses des grands hommes :

5 « [Nicole] avait vu de trop près M. Arnauld, M. Pascal, $\mathrm{M}^{\mathrm{me}}$ de Longueville, M. de Tréville et les autres jansénistes pour les admirer. Il m'en a dit certains faits qui me le persuadent, et effectivement celui qui a le plus d'esprit est nécessairement le plus petit admirateur " (p. 288-289).

6 C'est dans le même esprit qu'il rédige une espèce de compte-rendu de sa lecture des Pensées et plus particulièrement son analyse de l'argument $\mathrm{du}$ « pari ».

7 Pour l'abbé de Saint-Pierre tout se fonde sur le tempérament qui est défini par «la délicatesse des fibres du cerveau » et qui détermine la sensibilité. Or, à ses yeux, Pascal était manifestement d'un tempérament maladif: ses fibres étaient certainement trop « délicats » et sa sensibilité fut donc excessive. D'un tel tempérament on peut dire avec La Rochefoucauld que "l'esprit est souvent la dupe du cœur ", et c'est ce que notre abbé va démontrer dans l'analyse du "pari». En effet, pour lui, Pascal a mis ici son génie géométrique au service de l'enthousiasme et de l'illusion, - c'est-à-dire du jansénisme.

Raisonnons avec l'abbé de Saint-Pierre : imaginons que Pascal n'eût pas subi les effets de ce tempérament maladif : il aurait alors perçu la force des raisonnements en faveur de l'existence de Dieu et de l'immortalité de l'âme, raisonnements que l'abbé fonde sur une version très floue des définitions cartésiennes ${ }^{3}$ et sur une conception très humaine des qualités divines : Dieu est tout-puissant, juste et bienfaisant, donc... Dans le contexte de ce déisme philosophique, le "pari » se réduit à un argument solide mais banal en faveur du plaisir, de l'intérêt bien entendu et donc de la bienfaisance, qui est « l'essantiel » de la religion. L'abbé de Saint-Pierre détourne ainsi le "pari» au service du Dieu des Philosophe ${ }^{4}$.

9 Pouvons-nous dater ce texte? La définition de la religion raisonnable nous semble très proche de celle préconisée par Usbek dans les Lettres persanes (1721) ; l'allusion à la lecture de Montaigne par Pascal repose sans doute sur la publication de l'Entretien avec M. de Sacy par Desmolets en 1728 ; l'abbé de Saint-Pierre met en pratique la réforme pittoresque de «l'ortografe » qu'il proposait en 1730 ; enfin sa critique de l'argument des contrariétés nous semble suivre de près le texte de l'anti-Pascal de Voltaire (Lettres philosophiques, XXV remarques $n^{\circ} 3$ et 6). Ces indices internes suggèrent une date entre 1734 et 1743 . Par ailleurs, deux des manuscrits portent la mention « corrigé » : il semble donc fort possible, comme nous l'a suggéré $\mathrm{M}^{\mathrm{me}}$ Bottaro Palumbo, que l'abbé les a revus en prévoyant une deuxième édition de ses Ouvrages de morale et de politique, parus pour la première fois entre 1737 et 1740 .

10 Or, au tempérament maladif de Pascal, l'abbé de Saint-Pierre attribue sa "docilité " à l'égard des théologiens de Port-Royal, - timidité excessive qui aboutit à la « sotize ». Sur ce point son texte nous semble très proche des Pensées philosophiques de Diderot :

11 «Pascal avait de la droiture; mais il était peureux et crédule. Élégant écrivain et raisonneur profond, il eût sans doute éclairé l'univers, si la Providence ne l'eût abandonné à des gens qui sacrifièrent ses talents à leurs haines. Qu'il serait à souhaiter qu'il eût laissé aux théologiens de son temps le soin de vider leurs querelles; qu'il se fût livré à la recherche de la vérité, sans réserve et sans crainte d'offenser Dieu, en se servant de tout l'esprit qu'il en avait reçu, et surtout qu'il eût refusé pour maîtres des hommes qui n'étaient pas dignes d'être ses disciples! On pourrait bien lui appliquer ce que l'ingénieux 
La Mothe disait de La Fontaine : Qu'il fut assez bête pour croire qu'Arnaud, de Sacy et Nicole valaient mieux que lui » $\left(\mathrm{n}^{\circ} 14\right)$.

Ce texte de Diderot ne fut publié qu'en 1746: le rapprochement avec le manuscrit de l'abbé de Saint-Pierre pose un problème que les biographes ne permettent pas de résoudre, mais la question des sources de Diderot est notoirement complexe. Une version du texte de l'abbé de Saint-Pierre a fort bien pu circuler parmi les philosophes.

Enfin, ce compte rendu de l'abbé de Saint-Pierre met en évidence le scandale que constituait au XVIII siècle le refus pascalien des preuves naturelles et métaphysiques de l'existence de Dieu. Ce refus s'exprime fortement dans le chapitre XX de l'édition de PortRoyal des Pensées et il est pour ainsi dire souligné par l'effort maladroit de Nicole qui, dès 1670, donne en abrégé les preuves raisonnables auxquelles, prétend-il, Pascal aurait souscrit. Ce scandale allait fortement marquer la lecture philosophique des Pensées: d'Alembert et Condorcet opposeront les pensées religieuses et les pensées philosophiques; Chateaubriand s'effrayera : "Quel ne fût point devenu ce grand homme s'il n'eût été chrétien?»; Victor Cousin s'indignera et dénoncera violemment cette foi anticartésienne; dans la même tradition Lucien Goldmann réduira la foi pascalienne au " pari ». La lecture paradoxale de l'abbé de Saint-Pierre annonce en ce sens une longue tradition critique qui n'a été corrigée de nos jours que par le retour au texte de Pascal.

\section{Note sur les manuscrits de l'abbé de Saint-Pierre}

Il existe trois versions de ce texte parmi les manuscrits de Nauchâtel : mss R.141, R.157, p. 73 ss, et R.195, p. 70 ss. Les deux copies R. 157 et R.195 sont marquées « corrigé » et sont identiques. Cependant, en comparant ces différents textes, il nous a paru que le ms R.141 constituait une nouvelle et meilleure version du texte sauf pour les $\$ \S 12$ et 13 où le copiste a commis l'erreur d'un "saut du même au même ». En particulier, on remarque que le ms R.141 comporte trois paragraphes $(\$ \S 14,15,16)$ absents des autres manuscrits. Nous avons donc suivi le ms R.141 en signalant les variantes du ms R.157 pour les $\S \S 12$ et 13.

\section{«Pascal Écrivain des plus eloquans »}

\section{(ms Neuchâtel R. 141 et R. 157)}

je viens de relire avec plezir quelque chose des ouvrages de pascal. il etoit né d'une santé foible et delicate et par conséquant d'un tanperament plus sansible.

La delicatesse de son tanperament lui avoit ouvert l'esprit de si bonne heure dans son anfance que ses parans commancerent a craindre qu'il ne vécut pas longtems.

Cette délicatesse dans les fibres de son cerveau se trouva propre a lui fournir sur tous les objets des santimans plus delicats et plus distinguez; c'est pour cela que de bonne heure il aima la lecture de montagne auteur plein de ces santimans délicats, mais ou il y a peu de force et de justesse dans les rézonemans.

mais ce qu'il ne trouva pas dans montagne sur le rézonemant, il le tire de la geométrie ou il s'aplique dans sa premiere jeunesse avec un succez etonant. or d'un coté santir vivement et délicatemant, et de l'autre rézoner avec force et justesse, c'etoit tout ce qui etoit necessaire pour devenir ecrivain fort eloquant. aussi voyons nous dans ce que nous avons de lui ancore plus de cette grande eloquanse qui ebranle et terrasse les esprits du commun que de cette filozofie saje qui ne sonje 
qu'a convaincre l'esprit et qui est toujours en garde contre l'antouziasme et contre l'illuzion des santimans ${ }^{5}$. //

Personne ne peut mjeux prouver que pascal la verité de la maxime qui dit que l'Esprit est sauvant la dupe du cœur, c'est a dire que nous jugeons souvant selon nos craintes prézantes et selon nos dezirs prézans, et que sans nous en apersevoir, nos interets ou vrais ou aparans nous servent de preuves pour nos jugemans et malheureuzemant nos craintes prézantes, nos dezirs prézans ne sont fondez que sur des erreurs ou des opinions ou préjugez populaires qui n'ont pas eté examinez. cela me fait panser que plus on est sansible aux biens et aux maux, plus on craint les uns, plus on dezire les autres, et plus on est dispozé a parvenir a une grande eloquanse; c'est que la grandeur de l'eloquanse dépand particulieremant de la grandeur et de la vivacité des images des biens et des maux que produit la grande sansibilité.

Si pascal se fut borné a démontrer par rézon que l'essantiel de la religion cretienne de St mathieu 7.12 c'est la pratique de la charité bienfaizante pour plère a dieu, et qu'elle sufit pour en obtenir le paradis, il en fut aizémant venu a bout.

car il pouvoit aizémant démontrer par rézon qu'antre cette propozition il y a un paradis destiné aux bienfaizans et une punision destinée aux méchans aprez la mort, et celle ci, il n'y a point de paradis a esperer // pour les bienfaizans, ni de punision a craindre pour les malfaizans, la plus grande superiorité de vraisamblance est pour la premiere, et cette grande superiorité de vraisamblance lui auroit sufi pour démontrer que l'homme prudant doit agir en conformité pour augmanter son bonheur.

il pouvoit amprunter de Descartes la preuve de la distinxion des deux substances qui compozent l'homme, et prouver que les ames séparées des corps etoient aussi durables que le sont les corps qui dureront toujours.

il pouvoit tirer des méditations de ce filozofe de quoi montrer qu'il y avoit autant d'impossibilité que le corps fut sansible au plezir et a la douleur ou que l'ame fut divizible, qu'il y a d'impossibilité qu'un triangle soit quaré, ou qu'un quaré soit triangle ${ }^{6}$, et qu'ils peuvent subsister l'un sans l'autre séparémant.

De la nature de l'ame qui est plus noble que la nature du corps, il pouvoit conclure qu'il etoit beaucoup plus vraisamblable que les ames dureroient du moins autant que les corps qui dureront toujours et qu'ainsi la grande superiorité de vraisamblance etoit pour l'immortalité de l'ame et que pour la conduite la plus prudante des axions de la vie vers la charité bienfaizante, il n'etoit question que de connoitre qu'il etoit infinimant vraisamblable que l'Etre parfait etoit infinimant juste et bienfaizant. //

or de l'immortalité de l'ame c'est a dire de son existance perpetuelle de la durée perpetuelle de ses santimans et de ses idées, il pouvoit aizémant conclure ${ }^{7}$ que notre bonheur ou notre malheur aprez la mort dépandoit du bon uzaje de notre liberté, de nos bonnes ou de nos mauvaises axions, ou de notre bienfaizance ou de notre injustice.

De la perfexion des atributs de la divinité, c'est a dire de sa toute puissance, de sa justice et de sa bienfaizance infinie, il pouvoit aizémant conclure qu'il etoit plus vraisamblable que l'ame d'un trez méchant homme sera traitée diferamant aprez la mort que l'ame d'un homme trez juste et trez bienfaizant ${ }^{8}$. Ainsi voila la récompanse et la punision démontrées, ou du moins la superiorité de la vraisamblance pour l'imortalité de l'ame, et pour la justice et la bonté infinie de l'Etre parfait.

ainsi voila l'existance du paradis et de l'anfer démontrée quant a la superiorité de vraisamblance.

or $^{9}$ n'est ce pas là l'essantiel de la religion raizonable ? et que pouvoit faire de mieux pascal, que d'amploïer son eloquanse naturelle a peindre vivemant d'un coté la tranquilité, la douceur, les plezirs innosans et les autres agrémans de la vie prézante de l'homme juste et bienfaizant, et de l'autre les inquietudes les chagrins et les autres malheurs de la vie de l'homme injuste et méchant, et apostropher ansuite celui qui doute et qui délibere quelle conduite il tiendra pour arriver au 
plus grand bonheur dont il soit capable? quels biens immanses risquez vous de perdre? quels maux risquez vous de vous attirer a votre mort, si vous prenez la voye de l'injustice et quels biens immanses risquez vous d'aquerir en prenant la voye de la justice et de la bienfaizance?

cet argumant de prudance a une force infinie quand on a démontré la superiorité de vraisamblance tant pour l'opinion de // l'ame que pour la justice et la bonté de l'Etre parfait. Que j'ai de regret que Pascal eloquant comme il etoit, soit resté dans plusieurs des obscuritez de l'anfance de la raison particuliere ${ }^{10}$ au lieu de se contanter de démontrer eloquamant les veritez de prudance qu'il tenoit de la raizon universelle, c'est a dire de Dieu meme auteur de la rézon qu'il a donnée universelemant a tous les hommes.

Pascal n'a pas pris garde que la preuve qu'il tire de la grandeur et en meme tems des foiblesses de l'homme le plus parfait, pour prouver la necessité du pèché originel, n'etoit aprez tout qu'un trez mauvais argumant et un pur sofisme qui ne prouve rien sinon que l'homme n'est pas fait originairemant pour etre infini en perfexions, ni pour etre parfait de tout point, mais pour etre ${ }^{11}$ trez borné et par conséquant trez mèlé de perfexions et d'inperfexions .

Pascal etoit beaucoup plus écléré que ceux qu'il consulta, mais devenu plus habile qu'eux, il n'eut pas la sage hardiesse de comparer son esprit aux esprits des autres, et le tout vint de son tanperamant timide; ainsi il ne sortit point de la docilité de disciple lui qui devoit etre consulté comme maitre par les autres maitres moins eclérez; ainsi sa timidité ${ }^{12}$ excessive ou plutôt sa sotize lui fit prandre trez inprudanmant des hommes trompez par une autorité acordée sans Raizon a des gens qui n'avoient rien examiné pour des maitres trez // raizonables qui avoient tout examiné et qui etoient trez dignes d'etre crus sur leur parole.

il eut une grande maladie vers sa trantieme année cauzée par un excez d'aplication aux matématiques. Son esprit en fut afoibli et il ne songea plus guères qu'a la morale depuis cet afoiblissemant. il s'y trou va un peu neuf et par conséquant fort timide et mécaniquemant dispozé a recevoir docilemant les opinions de ces esprits afirmatifs qui ne font nul uzaje du doute et qui n'ont cepandant presque rien examiné de ce qu'ils ont reçu bonemant d'autres bons ancètres qui n'avoient de leur coté rien examiné de leurs plus anciennes tradisions : voila pourquoi il a eté simple pour s'abaisser jusqu'a recevoir quelques unes de leurs opinions absurdes en supozant qu'il $y$ avait une vraye grandeur d'esprit et qu'il etoit beau de soumettre quelquefois le rézanable a l'absurde.

\section{NOTES}

1. J. Drouot, L'Abbé de Saint Pierre, Paris, 1912, p. 35 : texte du ms Rouen 950 ; la référence pascalienne est omise de la version publiée (Rotterdam 1737), XIII p. 3-5.

2. Abbé de Saint-Pierre, Ouvrages de morale et de politique, vol. XII (Rotterdam, 1737), p. 86-87 : « Sur M. Nicole, le plus habile écrivain de morale de nos jours ». Voir Z. Tourneur, "Pascal "ramasseur de coquilles" : un contre-sens de Sainte Beuve ", Revue universitaire, 1934, I. 37-40, et Pascal, O. C., éd. J. Mesnard, vol. I (Paris 1964), p. 1030-31.

3. Voir aussi l'éloge de Descartes dans ses Euvres diverses (Paris, 1730) vol. I, p. 282 et dans ses Ouvrages de morale et de politique, vol. XI, p. 48ss.

4. Voir aussi dans les Ouvrages de morale et de politique le « Discours sur le dézir de la béatitude » (XI, p. 531 ss), les "Observations sur la béatitude après céte vie à Madame D.P." (XIII, p. 11ss), et la 
Proposition XXXII : "L'espérance la plus agreable et la plus utile aux particuliers et à la société, c'est l'espérance d'une segonde vie toujours délicieuze, destinée aux bienfaizants" (XIII, p. 373 ss).

5. ms R. 157 : qu'excitent les orateurs.

6. ms R 157 : qu'il y a d'impossibilité qu'une figure de triangle devienne un quarré ou qu'un figure de quaré devienne triangulaire en demeurant les mêmes.

7. ms R. 141: qu'il etoit plus vraisemblable que l'ame d'un trez mechant homme... [= saut du même au même].

8. ms R. 141 : phrase suivante omise.

9. ms R. $157: 3$ paragraphes omis.

10. ms R. 157 : humaine.

11. ms R. 157 : un Etre.

12. ms R. 157: sa timidité ou plutôt sa sotize lui fit prandre trez inprudanmant certains prezomptueux anthousiastes pour des maitres trez raizonables.

INDEX

Mots-clés : Pascal, Saint-Pierre (Abbé de), manuscrit

\section{AUTEUR}

\section{ANTONY MCKENNA}

Université de Saint-Étienne Institut Claude Longeon (CNRS UMR 5037) 